\title{
Are Deformities Stigmatizing? A Surgeon's Approach*
}

\author{
A. GRACE WARREN \\ Medical Superintendent, Hay Ling Chau Leprosarium, Hong Kong
}

\begin{abstract}
Disability in leprosy is often equated with the degree of obvious deformity. The patient's psychological approach to his disease influences his disability. The reconstructive surgeon can in many cases assist in rehabilitation by correcting stigmatizing deformities that constitute an obstacle to the patient's acceptance in the community.
\end{abstract}

The non-leprosy worker hears much about the disabilities that result from leprosy and comes to assume that every patient is excluded from normal social intercourse because of this disease. However, persons with marked disabilities of many types from causes other than leprosy appear to be fully accepted by their fellow men.

The leprosy patient is very self-conscious of any sign of the disease that, he feels, may advertise to others that he has or has had leprosy. His association with other leprosy patients and his own experience of the disease make him very aware of any small change in himself, and he assumes that everyone he meets will conclude that this change is due to leprosy.

Enquiry amongst mixed groups of young people, mainly students in Hong Kong, reveals that most of them have no real concept of the specific signs of leprosy. "The claw hand" is the commonest sign quoted, but this would only be recognized in its gross form and would probably include lesions from other causes that result in stiffening of the fingers or marked flexion in even a functional position. "Stumps and amputations" are also mentioned-but again no differentiation between surgical or accidental amputations could be made.

Much of the problem connected with stigma is in the minds of the patients themselves-who pass on to one another all the stories they know of people who have been rejected and ostracized, and assume that they face a real problem in trying to be accepted by society. Some will not even try to be accepted, but those who do are often very surprised. For example, a young man with five lesions of the borderline-tuberculoid type on his face was asked to see his ex-employer with a view to resuming his old job. The lesions, although fading, were still erythematous and visible; yet his employer was not concerned about the patches.

It would appear that although leprosy, "Ma Fung" as it is locally called, is still greatly dreaded by the older generation, it is feared less by those under the age of 30 who would not recognize the less severe deformities, and certainly do not worry about a disease called "leprosy" unless personally confronted with it by name.

* Received for publication, 14 February, 1972. 
Yet for many patients the presence of any obvious lesion of the skin, or other physical deformity, is an insurmountable obstacle to acceptance. For many of these, surgery will help the psychological problem, for they think that if they are questioned about the lesion, they can say they have had an operation or an accident, and show the scar as proof.

In other countries, the signs of leprosy are of different stigmatizing import. For example, in some areas of New Guinea it is a social custom to cut of fingers as a sign of mourning; the loss of fingers is therefore not necessarily connected with leprosy. In countries where people are greeted by placing the palms of the hands together in a "salaam" (e.g., Thailand), the presence of a crooked fif th finger is a real disability and the patient may ask for it to be straightened by arthrodesis, though not in a position of function, so that he may feel socially more acceptable. Social customs do influence the patient's attitude to the disease and must be given due weight when deciding on the course of treatment to advise. The same basic problems are present in all the countries of South-east Asia. However, their incidence varies in different countries; this may be due to racial differences in the host-parasite relation.

Some of the methods of correcting the common deformities are described by Antia and by Brand in Leprosy in Theory and Practice (Eds. Cochrane and Davey, 1964). Many other procedures are described in surgical text-books and journals; only a few references will be given in this paper. The most common deformities about which a surgeon is consulted are those affecting the face, the hands, and the feet.

\section{THE FACE}

Even if the patient has multiple deformities, he is usually mainly concerned about those on the face, since he feels they are the most obvious and hence carry the greatest stigma.

The surgeon who is interested in restoring a face to social acceptability must study the faces of the community from which the patient comes. He will be surprised at the variations present in a national group that may at first sight give the impression of uniformity of features.

(a) Nose. Deformity may vary from scars, due to ulceration, to total collapse, with or without loss of the columella. In lepromatous leprosy destruction of the septum is a comparatively late complication, but the skin of the nose is usually still intact and a post-nasal epithelial inlay allows the contour to be built up with the aid of a graft of bone or cartilage. In selected cases, an internal prothesis may be used instead of the bone graft. Although Crockett's method (Tovey, 1965) may save time, the two-stage procedure gives more reliable results in the hands of the occasional operator. From experience it may be affirmed that these procedures give a cosmetically acceptable result that provides permanent improvement in a patient in whom the disease is fully controlled. Mongoloid races usually ask for a straight nose but do not require a high tip, and a columella strut is therefore rarely required; the latter is more important for races with more prominent noses.

(b) Eyebrows. Partial or complete loss of the eyebrows is a common result of long-standing lepromatous leprosy in all races, and especially worries those who have bushy eyebrows. It is not a problem with Chinese women, who of ten pluck their normally scanty eyebrows. 
The method of replacement will depend on the quality of eyebrows required-thick ones require temporal artery island grafts, but scanty ones will often look more normal if replaced by individual hair follicles or punch grafts such as are now used for baldness of the scalp. In the Chinese, free graf ts of post-auricular hair-bearing skin provide soft, fairly thin grafts which are very acceptable (see Fig. 1). If the patient can be induced to wear heavy-rimmed glasses many people will never notice defects of the eyebrows, eyes or nose (Fig. 2).
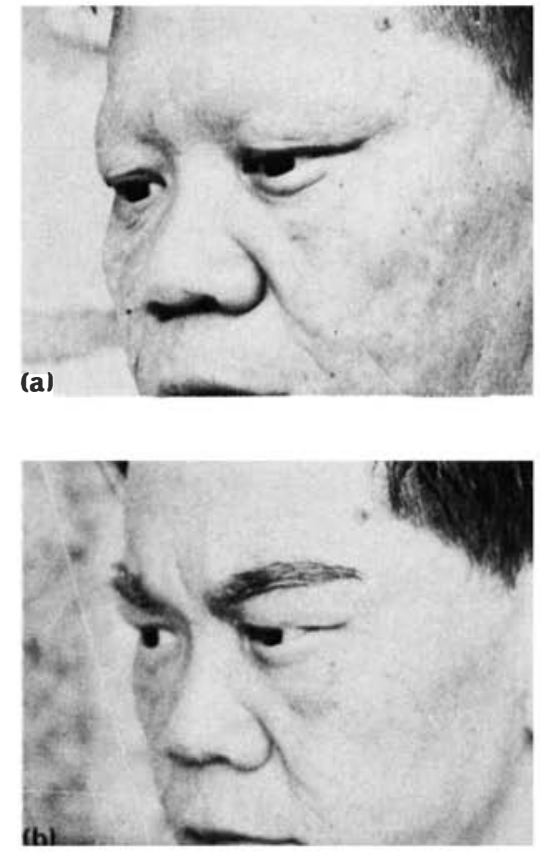

Fig. 1 (a \& b) Loss of eyebrows replaced by free grafts of hair-bearing post-auricular skin.
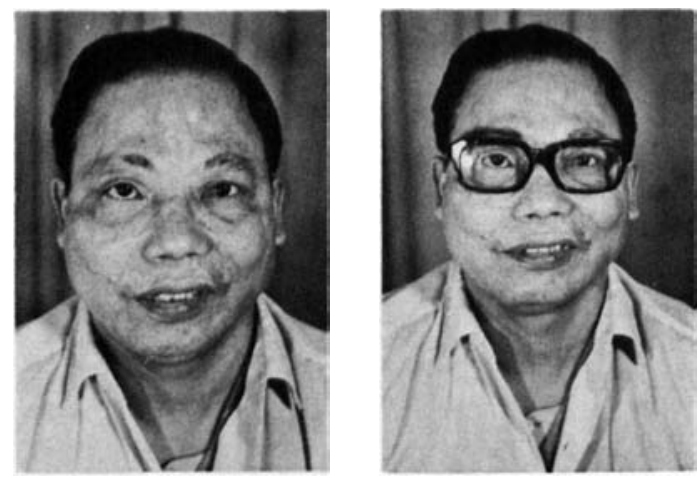

Fig. 2 Defect of eyebrows and right eye are not obvious with heavy-rimmed glasses. 
Although loss of eyebrow hair appears to be a universal complication of lepromatous leprosy, scalp baldness is not. Alopecia due to leprosy does, however, affect the Japanese, and was a real problem until wigs became popular and fashionable.

(c) Ears. Residual bagginess of the ear-lobes is easily trimmed by routine plastic measures, but such bagginess is not a stigma in Dyaks and other peoples who stretch the ear lobes (Fig. 3).
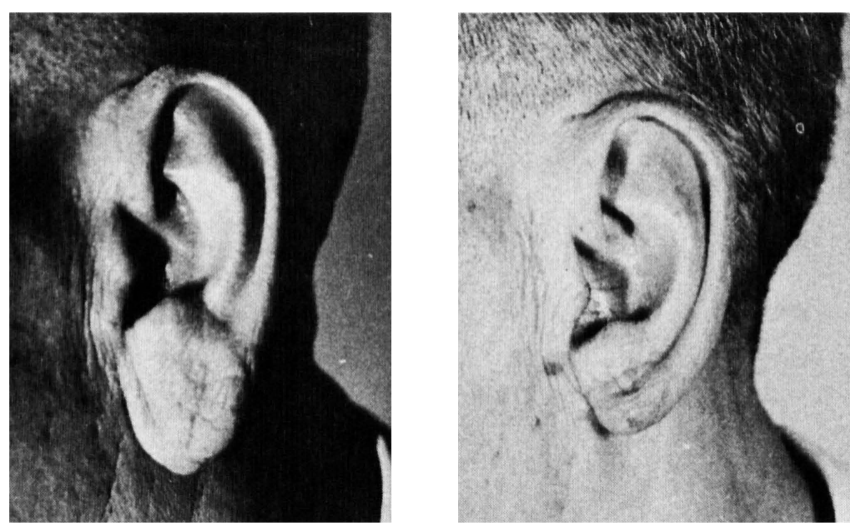

Fig. 3 Baggy ear lobes-before and after trimming.

(d) Facial palsy. This may often affect an eye and/or the mouth, and may be unilateral or bilateral. Facial paralysis from many causes is common in Asia, and many sufferers do not realize that anything can be done to relieve the inconvenience of constant dribbling from the mouth, and difficulty in eating and drinking. Constant watering of the eye and conjunctivitis are accepted as inevitable, and when ignored frequently result in blindness or diminution of vision.

Patients on whom temporal musculo-fascial transfers have been performed are generally grateful for the improvement in appearance and comfort, even if normal facial movements cannot be restored (Fig. 4). In the older age group, active muscle transfer may not be practical and in these patients with temporary paralysis of the orbicularis oculi, tarsorrhaphy will help preserve vision. Internal tarsorrhaphy (Warren, 1966) provides a more cosmetically acceptable procedure than lateral tarsorrhaphy.

Surgical procedures aimed at preventing blindness are a very important part of the surgical programme; it is not always possible to prevent diminution, or loss, of sensation, but the combined problem of blindness associated with loss of feeling in hands and feet should be forestalled.

(e) Facial skin scars are often difficult to remove, but the more severe ones can sometimes be improved. A face-lift may help to rejuvenate the prematurely aged. Patients develop an amazing trust and confidence in the surgeon and expect wonders to be performed. Their enthusiasm needs to be curbed! Deformities may also be due to non-leprosy causes; these may need to be dealt with before the patient can be confident that he will be socially accepted again. 

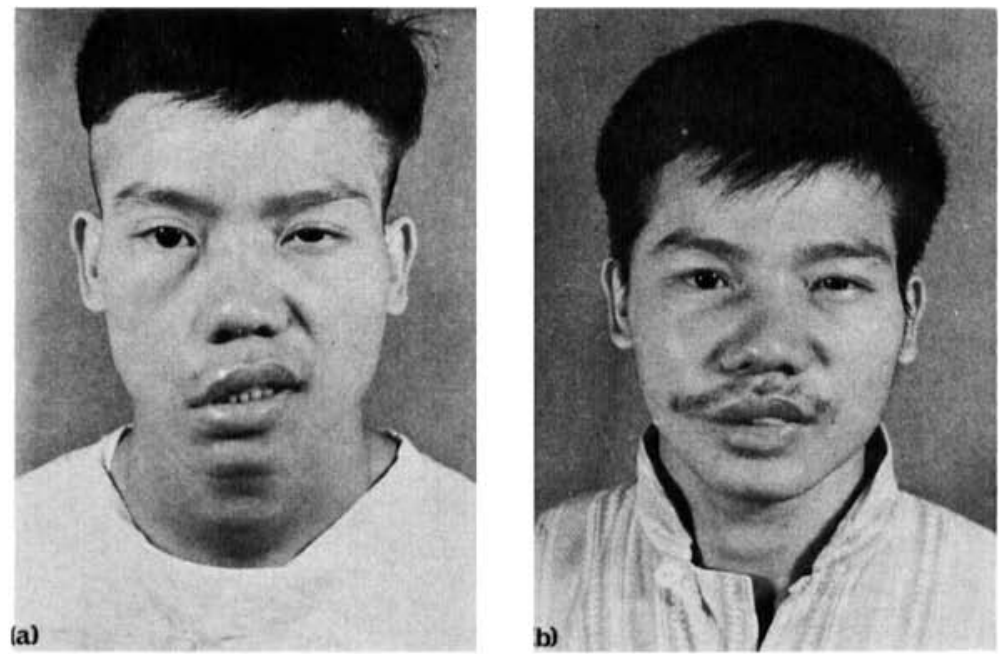

Fig. 4 (a) Facial palsy-notice drop of lip on patient's right side and increased width of palpebral fissure; (b) same face after use of temporal musculo-fascial transfer to the eye and a fascial slip to elevate the angle of the mouth.

\section{THE HANDS}

The commonest deformities for which correction is sought are loss of lumbrical function and loss of thumb opposition. These can be corrected by such routine methods as the Brand "many-tailed" procedure, using a wrist extensor as a lumbrical replacement and a sublimus tendon to provide opposition of the thumb. However, before any surgery is begun the hand should be carefully assessed for muscle power and mobility, and a surgical plan devised. Instability of the thumb metacarpo-phalangeal joint may result from ulnar-nerve involvement and may on casual examination appear as loss of median-nerve function. It can best be dealt with by an arthrodesis of the affected joint, providing a strong stiff thumb. The patient may request arthrodesis of the interphalangeal joint in these cases, but if this is done more disability may result. Flexion and extension of the interphalangeal joint of the thumb is a very useful function and should be maintained if possible.

Even in the replacement of a lumbrical loss, consideration should be given to the patient's use of his fingers. A lumbrical inserted on the ulnar side of the index finger may provide good adduction and so improve the ability of the Indian patient, for example, to pick up rice balls, but will be a disadvantage to the Chinese patient who needs to abduct the index finger to use chopsticks. Hence in Chinese patients the index lumbrical should be placed on the radial side of the finger. "Claw hand" is stated to be the most easily recognized stigma, but few people seem to differentiate between the true claw hand, resulting from median and ulnar paralysis, and fixed flexion deformities from other causes. Many patients with badly deformed hands have no desire for reconstructive surgery, since they have learnt to cope with them as they are; this would suggest that at least for them the stigma is no great problem. 
However, patients do present asking for surgery for what we may think are minor problems-crooked fingers, enlarged nerves on the hands, and wasting of the hands due to atrophy of small muscles. Chinese leprosy patients are very conscious of wasting of the muscles of the thumb web; the cavity thus formed is known as "Tiger's mouth". Methods of filling out the space are sought and many patients are seen who have had a paraffin injection into the space. This frequently results in a hard mass which contracts and tethers the thumb in the adducted position, and this mass may have to be removed before any real reconstruction can be attempted. Contracture of the thumb-web from any cause limits abduction of the thumb; to achieve a full range of movement, a skin graft is of ten desirable, but this is frequently refused as the patient feels the graft is more stigmatizing than the limited movement of the thumb, and one may have to be satisfied with doing a Z-plasty to deepen the web and give a little more range of movement (Fig. 5).

Dermofat grafts have been used to fill out the space, but many of these are absorbed after a few years and the deformity recurs; or else they become small
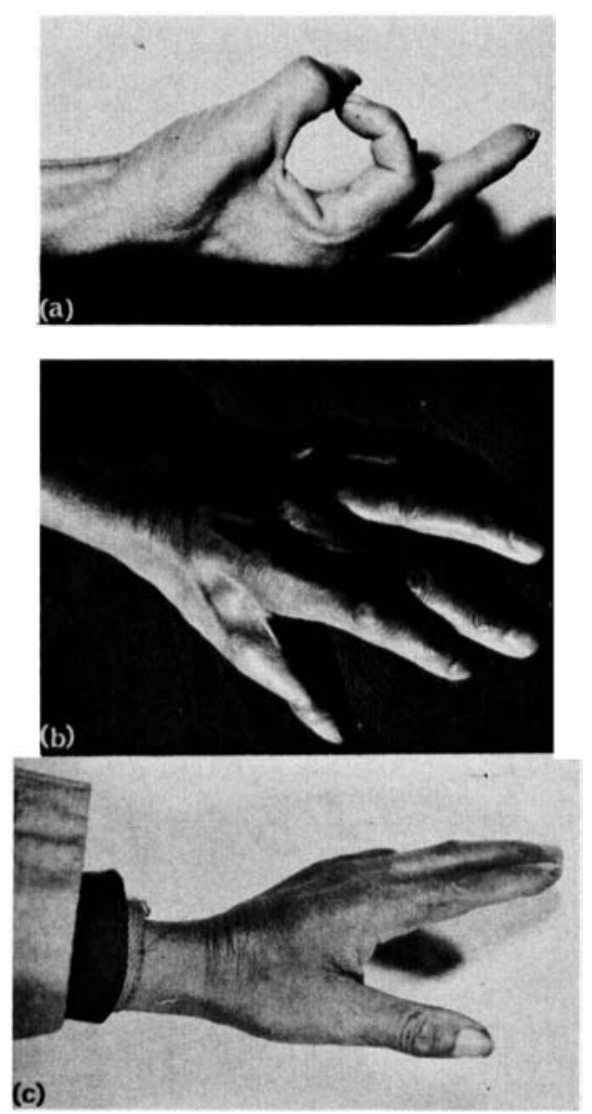

Fig. 5 (a) Typical "Tiger's Mouth" due to atrophy of small muscles of thumb web; (b) pellet that has resulted from use of Ivalon to fill out space; (c) web filled with Elicon. 

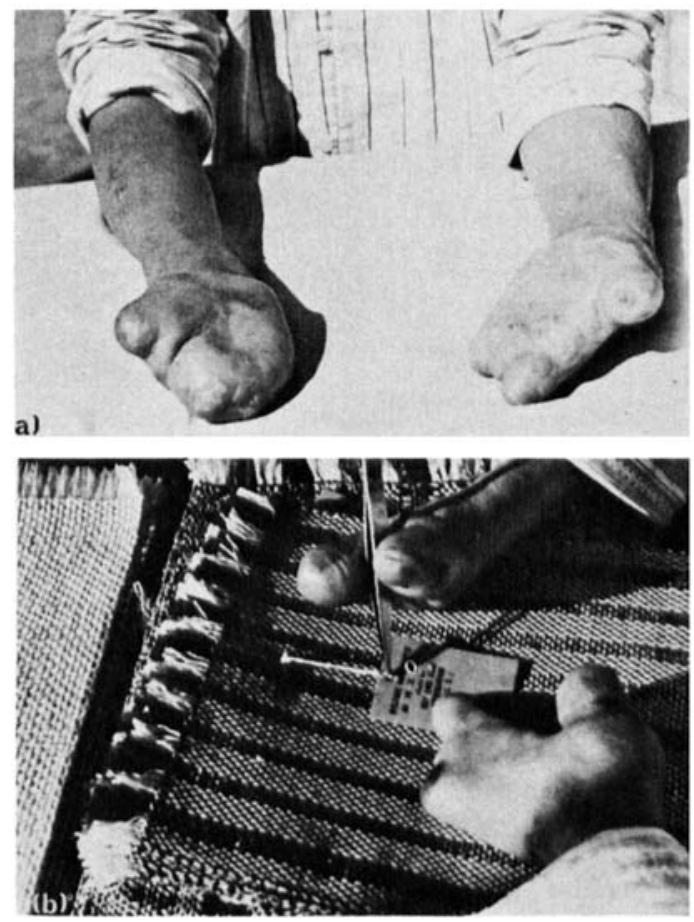

Fig. 6 (a) Marked disability from loss of all fingers; (b) regained ability. After removing a metacarpal from each hand, patient learnt to weave and do other hand work.

hard masses. Plastic and silicone materials have been tried, but again, some of these tend to form hard pellets. The ideal material must remain soft, be non-reactive, and permanent. The nearest approach to this is a Japanese material, Elicon, which we have been using for the past 6 years.

Most patients, unless they are resident at a centre where a considerable amount of reconstructive surgery is done, have little or no idea of the possibilities and are very hesitant before allowing their hands to be operated on, until unfortunately the se have become almost useless. Those who have seen what can be done come to expect miracles, and believe that any crooked finger can be straightened and restored to normal function.

Sometimes it is a matter of increasing the deformity in order to decrease the disability, as in the case of complete, or almost complete, loss of the length of all the fingers and the thumb. This leaves a stump of a hand that cannot by itself hold anything. Removal of one or more metacarpals will create a cleft that changes the stump into a two-finger hand-or "lobster hand" (Bunnell, 1944)-with which a patient can feed and dress himself and may learn to write, weave, or do other handwork [Fig. 69(b)].

\section{FEET}

Very few patients spontaneously present themselves seeking removal of stigma of the feet, probably because much can be hidden by shoes and trousers. They do 
not seem to worry about dropped feet as long as they can get around, and rarely seek help until marked disability from ulceration and infection has occurred. They dislike wearing toe-raising springs as they feel these will advertise their disease, though once admitted to a sanatorium or hospital they will acknowledge that the spring does make walking easier. In leprosy, the feet frequently become deformed to a degree where normal shoes cannot be worn without causing more ulceration. Special orthopaedic footwear may be ordered, but here in Hong Kong, and in some other countries, this footwear will not be worn as the patients feel it advertises their disease. Hence we have adopted the routine of remaking the feet so that normal soft shoes can be worn. "Make the foot fit the shoe-not the shoe to fit the foot." In this way a period of 3 to 6 months' hospitalization allows for healing of ulcers and surgical reshaping of the feet so that the patient is able to go home in soft-topped shoes with resilient rubber soles that can be bought "on the street". They are easily replaced and are not as expensive as orthopaedic shoes would be, even if these were available and acceptable. Sandals are not acceptable in this area, but a wide range of soft-topped shoes is available, and these have proved very satisfactory and are socially acceptable.

It is interesting to notice how attached patients are to deformed toes, even when these are of no functional use whatsoever and are constantly being traumatized by the footwear. They are most hesitant to give permission for amputation, although this would frequently result in a foot that is much more easily cared for. But many patients do request the amputation of a problem foot-if they have no other deformity-as they feel that in this way they are removing what stigma they themselves recognize. It is often difficult to assess the advisability of such an amputation, since the calf and even the thigh may be anaesthetic; the fitting of a prosthesis is thus a real problem.

\section{Conclusion}

The reconstructive surgeon who seeks to aid leprosy patients will never be without a challenge to his ingenuity. There are about half a dozen main basic reconstructive procedures which when mastered will meet the requirements of some 70 to $80 \%$ of leprosy patients. The remainder will set the surgeon exploring the literature for new methods and techniques to solve problems of the individual-loss of the helix, loss of the columella nasi, contractures of wrist in flexion or fingers in extension, clawed toes and inverted heels-yet the patients develop a real faith and trust in one who tries to help, and often take failures in good part, realizing that some are inevitable. Though some seem to expect to be helped and never express gratitude, many are most profuse, and even overwhelming, in their thanks and some come back years later with a thank-offering to let you know they are still well and the deformity has not recurred. As a group, one could not hope to find more grateful patients. The greatest thanks one can receive is to see a patient who was previously afraid of the world, going back and making good, remembering to care for his hands, feet and face, so that he keeps them in as good condition as possible and so obviates the necessity of further reconstructive or salvage surgery. 


\section{Ref erences}

Antia, N. H. (1964). Reconstructive surgery of the face, in Leprosy in Theory and Practice. 2nd ed. (Eds. Cochrane and Davey), p. 497. Bristol: John Wright \& Sons.

Brand, P. W. (1964). Operative techniques, in Leprosy in Theory and Practice. 2nd ed. (Eds. Cochrane and Davey), p. 483. Bristol: John Wright \& Sons.

Bunnell, S. (1944). Surgery of the Hand. 3rd ed. London: Pitman Medical Publishing Co. Ltd. Tovey, F. I. (1965). Reconstruction of the nose in leprosy: Lepr. Rev., 36, 215.

Warren, A. G. (1966). A method of medical tarsorrhaphy for correction of lagophthalmos and ectropion. Lepr. Rev., 37, 217. 